\title{
Dynamic Cities - Ciudades Dinámicas: Una propuesta simbólica para un mundo caótico
}

\author{
Martínez-Medina, Andrés \\ Universidad de Alicante, Departamento de Expresión Gráfica y Cartografía, Alicante, España \\ andresm.medina@gcloud.ua.es
}

\begin{abstract}
Resumen
Dentro de las actividades culturales de la 15 $5^{\text {a }}$ Muestra de Arquitectura de la Bienal de Venecia (2016) figura el programa "Time, Space, Existence" al que ha sido invitado el profesor de la Universidad de Alicante Joaquín Alvado Bañón con una instalación titulada Dynamic Cities. Este montaje se integra en la serie de exposiciones del European Cultural Center dentro del palacio Bembo. Se despliega una maqueta de dos dimensiones (y un vídeo) con una maleta abierta en todas sus tapas, ejecutada con un bastidor metálico y mostrando el equipaje: un total de doce tablas de piedra natural de distintos tamaños y formatos rectangulares de cantos curvos. El resultado es un collage de piezas pétreas atravesadas por sus huecos redondos que se ajustan como piezas de un puzle sobre un engranaje de barras.
\end{abstract}

Según el propio autor, con esta propuesta trata de demostrar la inestabilidad del mundo y, por ende, de las ciudades y las sociedades que lo habitan. Parte del principio del "efecto mariposa", que está en el origen de las teorías del Caos, e intenta establecer nexos entre muy distintas variables para sentar las bases de la innovación entendiendo la ciudad como un organismo cinético, no predecible como mecanismo, en donde la forma física es meramente el soporte de las actividades, las energías y los flujos de información. Se trata de una aproximación conceptual a la ciudad como un ecosistema abstraído hacia dos elementos: el vacío y el sistema dinámico. La instalación expresa simbólicamente la interacción entre los espacios públicos y las arquitecturas privadas como una metáfora de la Historia de las ciudades. Es una instalación que apuesta por los seres humanos, definidos por su condición racional que se muestran en su condición emocional y que acusan su condición social, condición que se favorece las ciudades.

Palabras clave: Ciudades dinámicas, Bienal Venecia, instalación artística, collage urbano, concepto simbólico.

\begin{abstract}
Within the cultural activities of the 15th Venice Biennale - Architecture Exhibition (2016) figures the program "Time, Space, Existence" to which has been invited the Professor of the University of Alicante Joaquín Alvado Bañón with an installation entitled Dynamic Cities. This Assembly fits into the series of exhibitions of the European Cultural Center within the Bembo Palace. It is displayed a model of two dimensions (and a video) with a suitcase opened in all caps, executed with a casting metal and showing the luggage: a total of twelve tables of natural stone of different sizes and formats rectangular of edges curved. The result is a collage of stone pieces pierced by their round holes that fit like pieces of a puzzle over a bar gear.

According to the author, this proposal tries to show the instability of the world and, hence, of the cities and societies that inhabit it. He parts of the beginning of the "butterfly effect", which is at the origin of the theories of Chaos, and attempts to establish links between different variables for laying the foundations of innovation and understanding the city as a kinetic, not predictable agency as a mechanism, where the physical form is merely the support activities, energy and information flows. It is a conceptual approach to the city as an ecosystem abstracted into two elements: the vacuums and the dynamic system. The installation express symbolically the interaction between them spaces public and the architectures private as a metaphor of the History of the cities. It is a facility that committed by human beings, defined by its rational condition shown in his emotional condition and who accuse their social condition, condition that is favoured by the cities.
\end{abstract}

Key words: Cities dynamic, Venice Biennale, installation art, collage urban, symbolic concept. 


\section{La propuesta-instalación de las Dynamic Cities}

Dentro del programa de actividades culturales que despliega en su entorno la 15 Muestra de Arquitectura de la Bienal de Venecia, figura el programa “Time, Space, Existence - Tiempo, Espacio, Existencia”. Este lema parafrasea el título del libro de Sigfried Giedion Tiempo, Espacio y Arquitectura, de 1939, el cual se erigió en el manual dogmático de la crítica operativa de la vanguardia del 'movimiento moderno' en arquitectura (ca. 1919-39) que pretendía cambiar el mundo haciendo tabla rasa y empezando de cero: una nueva arquitectura construiría una nueva ciudad que daría cabida a una nueva sociedad. El libro daba luz al principio de un camino, previsible y determinista, que se prometía revolucionario en sus inicios y que, medio siglo después, había dejado de brillar y solo traslucía sus sombras: la seguridad racional había sido sustituida por la incerteza emocional. Quizás porque la arquitectura por sí sola no era suficiente para cambiar el mundo y requería del apoyo y la complicidad con la propia 'existencia' humana, de aquí esta nueva reformulación de un título que reclama para la Arquitectura (el término ausente del eslogan) la totalidad de la trilogía "Tiempo, Espacio y Existencia".
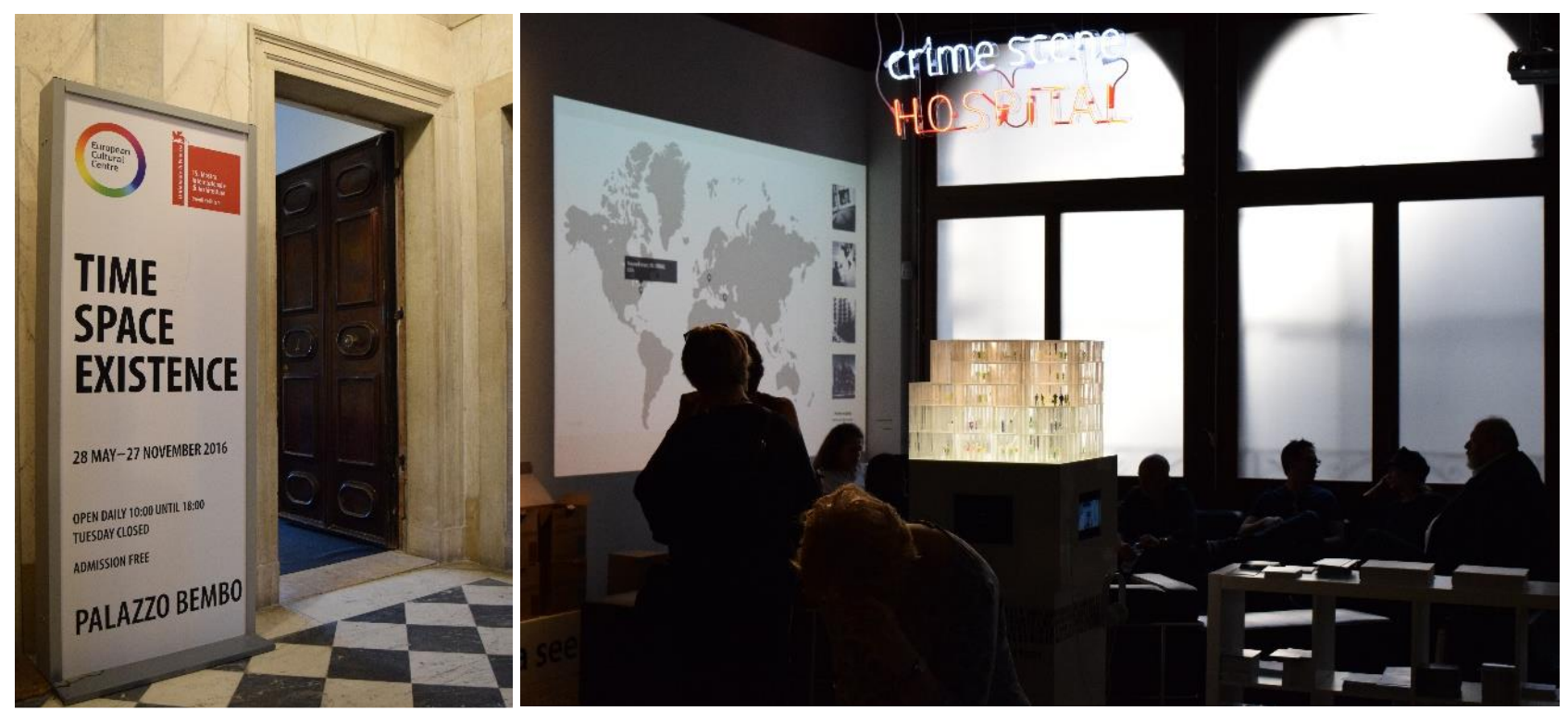

Fig. 01: Entrada al Palazzo Bembo y sala de debates y encuentros en su interior

Mientras los eventos principales de este acontecimiento se desarrollan en los recintos y pabellones oficiales de la Bienal de Venecia (El Arsenal y los Jardines de la Bienal), el programa de exposiciones organizadas por el European Cultural Center se despliega en tres palacios: Mora, Bembo y Rossini, todos ellos dentro de la trama densa y compacta de la ciudad de Venecia, emplazados en una ruta a pie que los vincula del lado norte del Gran Canal. En estas sedes solo se puede participar por invitación expresa de la organización. Este es el caso del profesor de la Escuela Politécnica Superior de la Universidad de Alicante (UA), Joaquín Alvado Bañón, quien fue seleccionado para montar su trabajo en el palacio Bembo junto a Auckland University of Technology and University of Sydney (AUS); ETH Zürich (CHE); MIT (USA); Nanjing University (CHN); Platform for Architecture + Research (USA); University of Melbourne (AUS); Royal Institute of Dutch Architects (NLD); School of Architecture of the Chinese University of Hong Kong (CHN); Sweet Sparkman Architects and University of Florida College (USA); Universidad Anáhuac México (MEX); Universidad Iberoamericana (MEX); Universidad Técnica Particular de Loja (ECU) y School of Architecture Tsinghua University (CHN). En el catálogo de esta singular exposición (isbn: 978-94-9078422-5) la instalación Dynamic Cities queda al lado de maestros de la talla de Denise Scott Brown, Peter Eisenman, Fumihino Maki o Jacob+Macfarlane.

En la primera de estas tres sedes, el palazzo Bembo, se ubica la sala de debates (Fig. 01) en torno a la cual giran las distintas estancias del histórico edificio donde se despliegan los stands y los montajes de los profesionales e instituciones invitadas. Entre lo más destacado estarían las propuestas que exploran la movilidad urbana 'limpia', la construcción sostenible, los estudios tipológicos de viviendas (sus variantes más que sus constantes), la arquitectura virtual (panorámicas de horizontes metropolitanos) y la compatibilidad del patrimonio arquitectónico con los equipamientos del futuro, entre otros. En la segunda de las sedes, el palazzo Mora (Fig. 02), abundan más las instalaciones artísticas (fig. 03) que las estrictamente técnicas de investigación y exploración (Fig. 04). Arquitecturas y artefactos sensibles que reaccionan ante la presencia humana; montajes y maquetas que cuestionan la escala y la materialidad de objetos y espacios; proyectos que proporcionan experiencias en la creación de ambientes virtuales; esculturas que se preguntan sobre la estabilidad de muchos principios científicos; instalaciones de vídeo-arte 
que deforman la realidad y movilizan resortes y recursos... En resumen, un amplio despliegue -más que de proyectos y propuestas que reafirmen las certezas - de auténticos montajes, maquetas y pantallas que nos cuestionan sobre la necesidad de no establecer límites fijos, ni lemas encorsetados, ni dogmas inamovibles: todo debe pasar por el tamiz de la experiencia humana concreta por lo que los presupuestos y fórmulas universales pierden validez y requieren del matiz de la Existencia. Frente a lo estático de la certeza emerge lo dinámico de la duda.

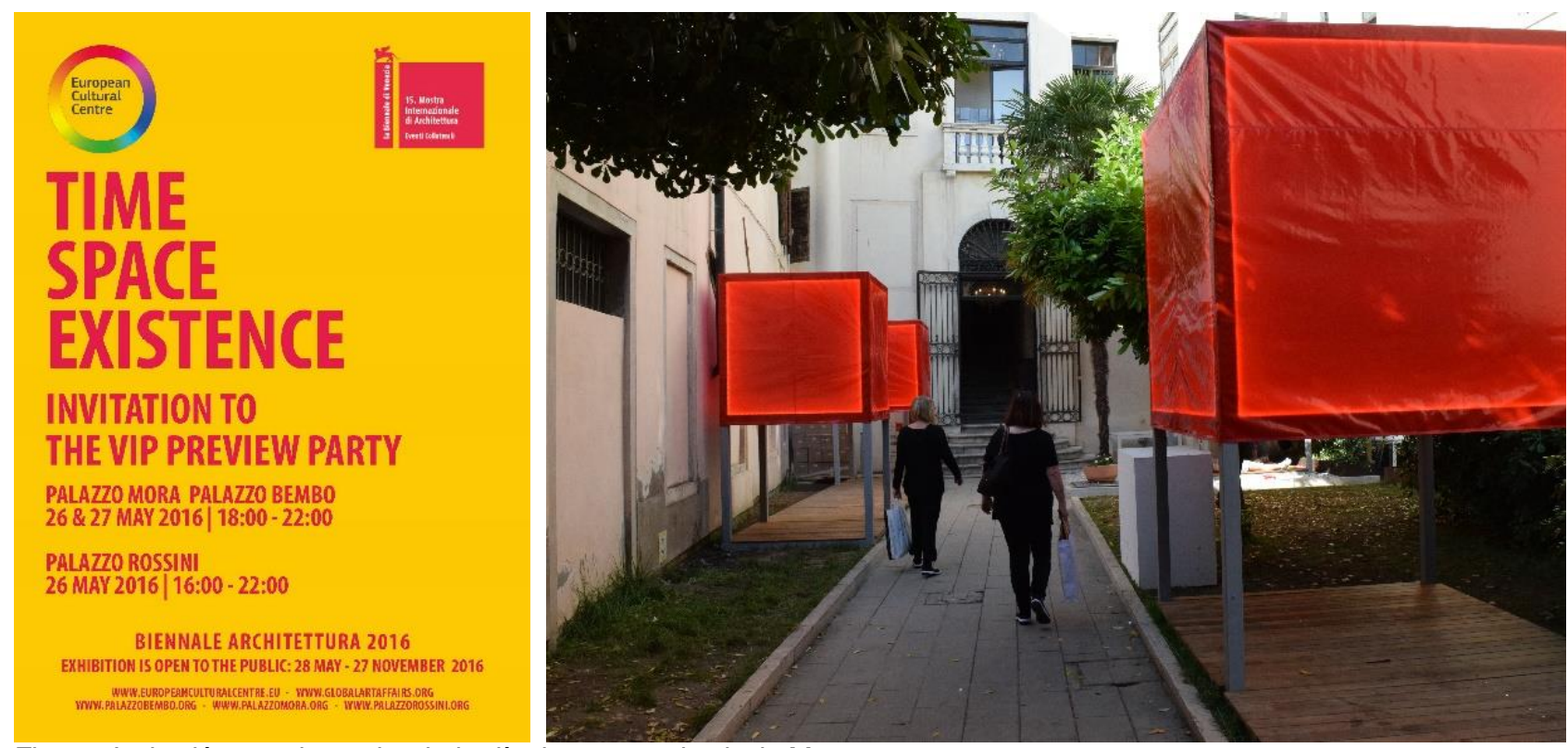

Fig. 02: Invitación para los palazzi y jardín de acceso al palacio Mora
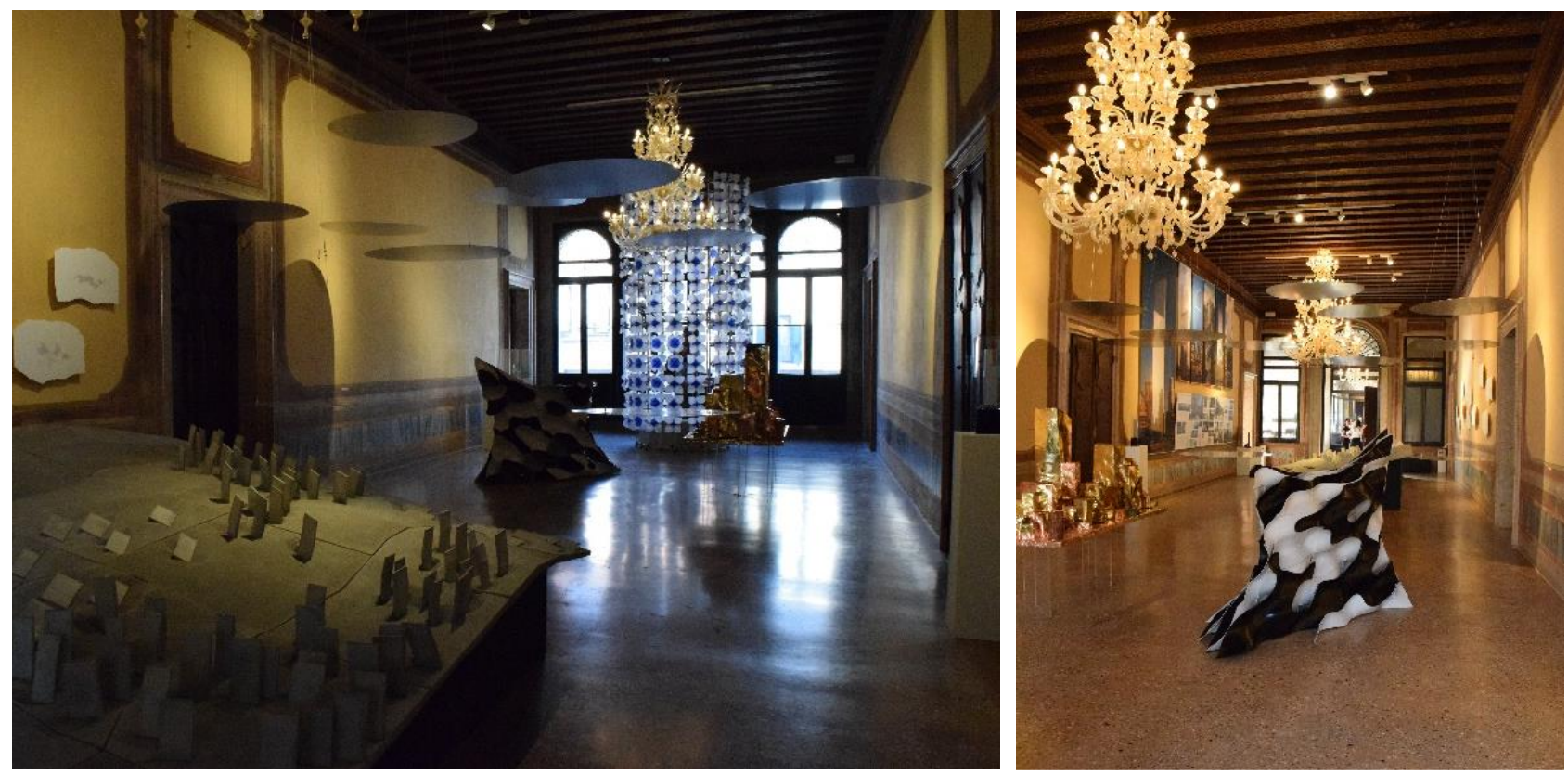

Fig. 03: Dos vistas del salón principal del palacio Mora con instalaciones artísticas 

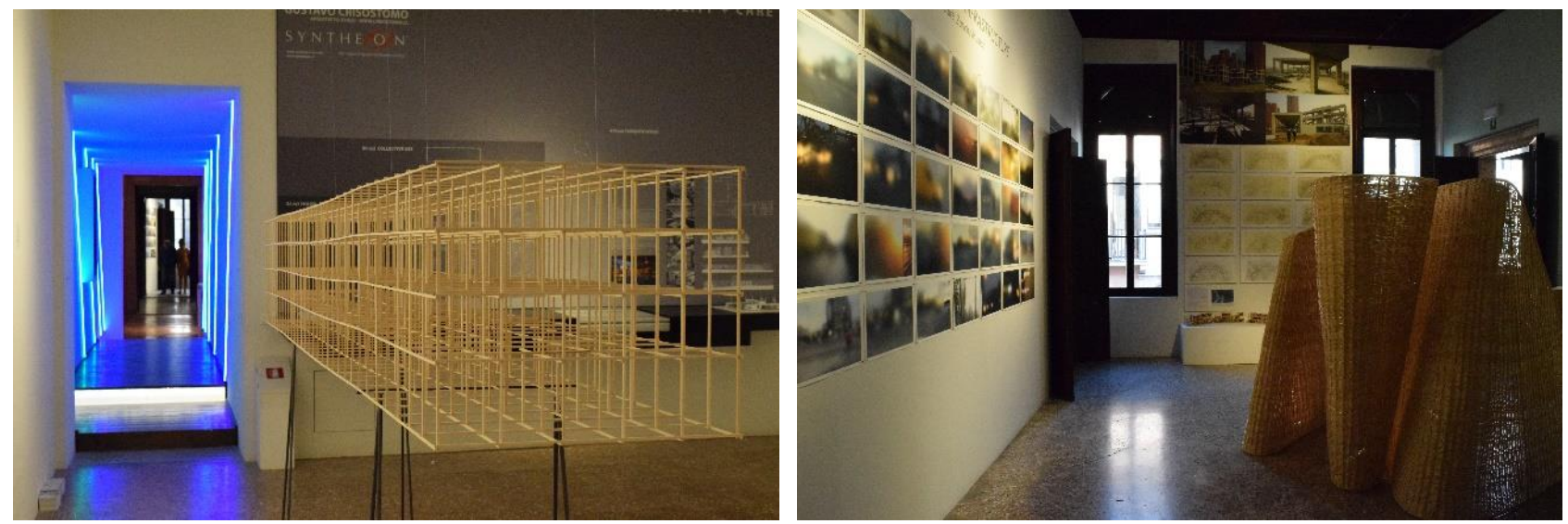

Fig. 04: Instalaciones que anteceden y acompañan a la instalación de Joaquín Alvado

En una de las salas del palacio Mora, lugar de paso obligado entre unos salones y otros, se sitúa la instalación del arquitecto y profesor de la Universidad de Alicante Joaquín Alvado Bañón (Fig. 05); un proyecto que ha contado con diversos apoyos institucionales, de patrocinios privados y, sobre todo, de un amplio equipo de jóvenes profesionales, en su mayoría egresados de la titulación de Arquitectura de la Escuela Politécnica Superior de la UA (ver detalle más adelante). El montaje de la propuesta de este profesor, una maqueta de dos dimensiones acompañada de la proyección de un vídeo ocupa toda la pared de uno de los muros de la sala y consiste en una maleta abierta en todas sus tapas (una especie de sobre para cartas desplegado: un rectángulo inscrito en otro rectángulo mayor), toda ella ejecutada con un bastidor de tubo metálico sobre la que se extienden los distintos elementos que integran el equipaje: hasta un total de 12 tablas de diferentes piedras naturales (con predominio de los mármoles) de distintos tamaños con formato rectangular y cantos redondeados — cerca a la proporción de los antiguos televisores-; cada una de estas piezas está taladrada por círculos de hasta tres diferentes radios y con entre 1 y 4 agujeros por 'pantalla' (Fig. 06). El resultado aparente es un collage de doce rectángulos pétreos, con una geometría suavizada en sus esquinas curvas y en sus redondos vacíos interiores, que intentan ajustarse como piezas de un puzle sobre un engranaje de barras metálicas. Esta instalación-maqueta tiene por título Dynamic Cities.

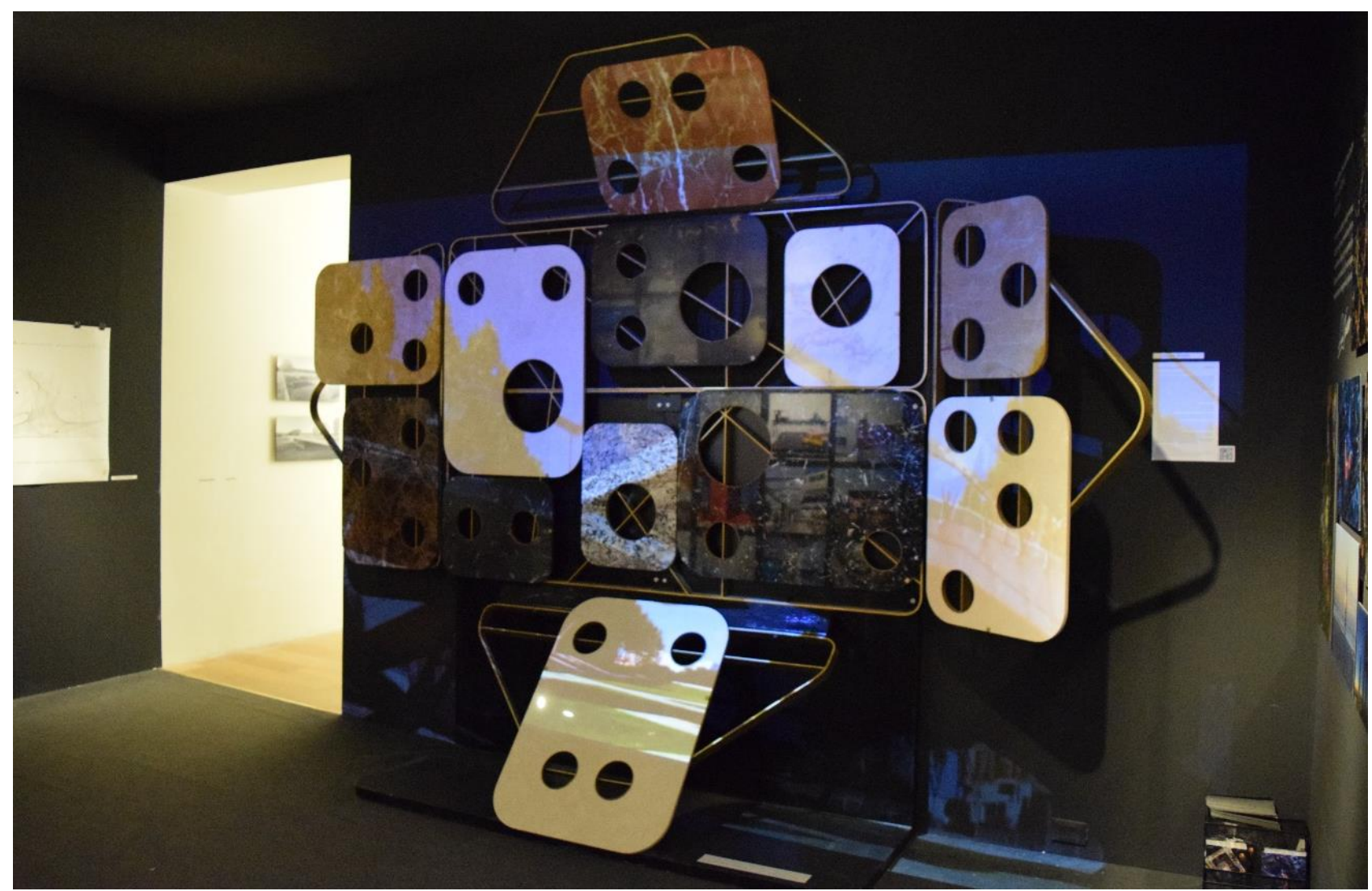

Fig. 05: Vista completa de la instalación Dynamic Cities con la proyección de video 
Según las palabras del propio profesor, con esta propuesta trata de demostrar la inestabilidad del mundo y, por ende, de las ciudades y las sociedades que lo habitan. Parte de un principio: "el efecto mariposa", que está en el origen de las teorías del Caos (Caos que tiene sus propias leyes —complejas — y su propio orden —inestable-) e intenta establecer nexos entre muy distintas variables para sentar las bases de la innovación. Entre las incógnitas que participan en esta ecuación se encuentran factores como sociedad, naturaleza, crecimiento, tiempo, cambio, sostenibilidad, tiempo, atmósfera, agua, adaptabilidad, bienestar y medio ambiente. Más que atender a parámetros medibles y mensurables racionalmente, se incorporan variables que conviene cuantificar cualitativamente para la mejora del hábitat y de las metrópolis y, por extensión, mejorar las condiciones del planeta. Porque este profesor (como la mayoría de los actuales pensadores sobre los fenómenos urbanos, aunque procedan de las múltiples disciplinas y campos de conocimiento que los abordan) coincide en un entendimiento de la ciudad como un organismo cinético, no predecible como mecanismo, en donde la forma física es meramente el soporte de las actividades, las energías y los flujos de información (lo que Françoise Ascher denominaría el sistema B.I.P.), todo ello en interacción con el medio ambiente y la naturaleza que las rodea y da soporte a su 'vida'.

Así pues, se trata de una aproximación conceptual a la ciudad como un ecosistema abstraído hacia dos elementos: el vacío y el sistema dinámico (Fig. 07). El vacío supone la iteración de los fenómenos, mientras que los sistemas dinámicos se relacionan con la sensibilidad de las condiciones iniciales. Estos vacíos (repartidos por las diferentes tablas de piedra) representan los espacios democráticos donde "se proyecta un conflicto entre el espacio virtual y el real", intentando trascender la concepción de la arquitectura como objeto estático para hibridarlo de experiencias, sensaciones y emociones. Los sistemas dinámicos hacen referencia a lo cambiante de las condiciones y las situaciones que convienen mapear — cartografiar - y cuyos diagramas se convierten en "estructuras dinámicas, tridimensionales". La instalación intenta expresarse de un modo simbólico de manera que muchos de los elementos dispuestos (forma, dimensión, disposición, geometría, etc.) asemejen piezas 'informales' que tienen la capacidad de ser "soportes estructurales del espacio". La interrelación e interacción entre los espacios públicos y las arquitecturas privadas se refleja como una metáfora de la historia de las ciudades, en donde muchas veces se han relegado las estructuras culturales que son las que dotan de sentido, vida y emoción a la propia Historia de la Humanidad.
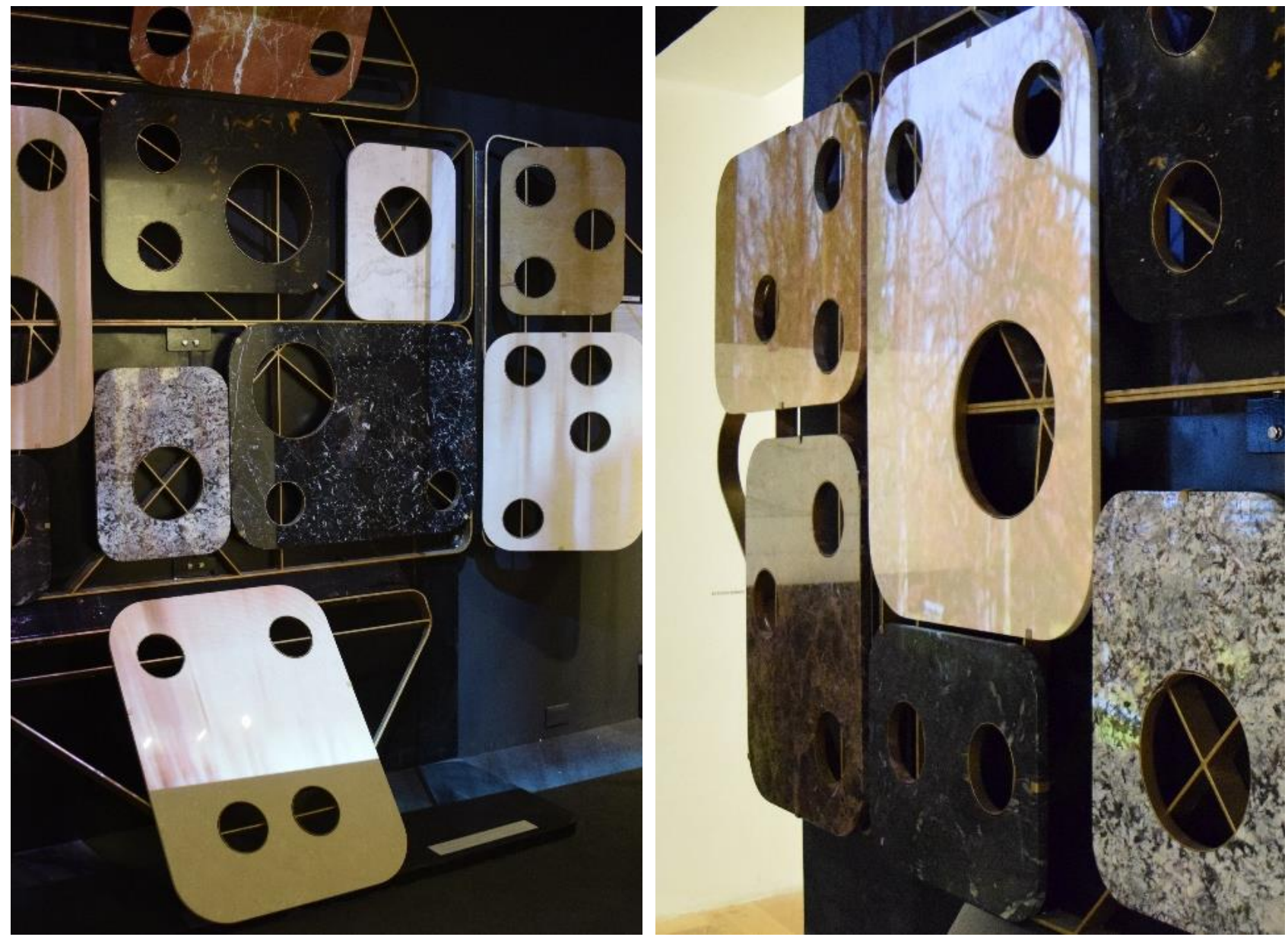

Fig. 06: Vistas parciales de la instalación Dynamic Cities con la proyección de video superpuesta 
En síntesis, nos encontramos ante una propuesta que apuesta por los cambios necesarios en los espacios públicos urbanos desde una posición no estática, sino atenta a los acontecimientos y a las condiciones cambiantes de la vida. Los seres humanos, caracterizados por su condición racional — geometría TV—, muestran en esta instalación su condición emocional — geometría circular- y, por extensión, los seres humanos acusan su condición social de seres enredados y conectados —-bastidor metálico de la maleta-, condición que solo es posible ejercer en las ciudades y en las metrópolis de nuestro pequeño planeta. Una última mención donde la diversidad y la condición internacional se hacen patentes, y que subyace en el discurso del montaje, está en la presencia, dentro del equipo humano, de diferentes egresadas y egresados de la Universidad de Alicante que se encuentran repartidos por los diversos continentes, lo que reafirma la voluntad de compromiso social sostenible de esta institución por encima de fronteras y geografías.

\section{Ficha Técnica de la Instalación}

Dynamic Cities. La Biennale di Venezia 2016

Exhibition Design: Angel Rocamora, Raquel Gonzalez

Video edition: Patick Vazquez, Vicente Pascual. Media Lab: UA

Video Tracks and Texts: José Antonio Carrillo (Dubai), María Javaloyes (Oasis Mhamid), Mary Pepa Ramos and Eduardo Landete (Düsseldorf), Anabel Ruiz (Alicante), Francisco Colom (Baku), Juana García Ladrón de Guevara (Madrid,) Jorge Almazán (Tokyo), Víctor Llanos (Barcelona), María Elia Gutierrez, David Fontcuberta and Paula Villar (Lima), Tomás Amat (Hong Kong).

Book "Dynamic Cities" Layout: Francisco Colom.

Institutional Support: University of Alicante: Paqui Milán, Elia Gutiérrez, Andrés Montoyo. Architectural Association Alicante: Vicente Castillo.

Exhibition Structure. Making of: Levantina S.L. - The natural stone company

Sponsorship: Main: Levantina - The natural stone company

Others: Tecnocemento, Guia Consultores, Cemex, University of Alicante, EPS.

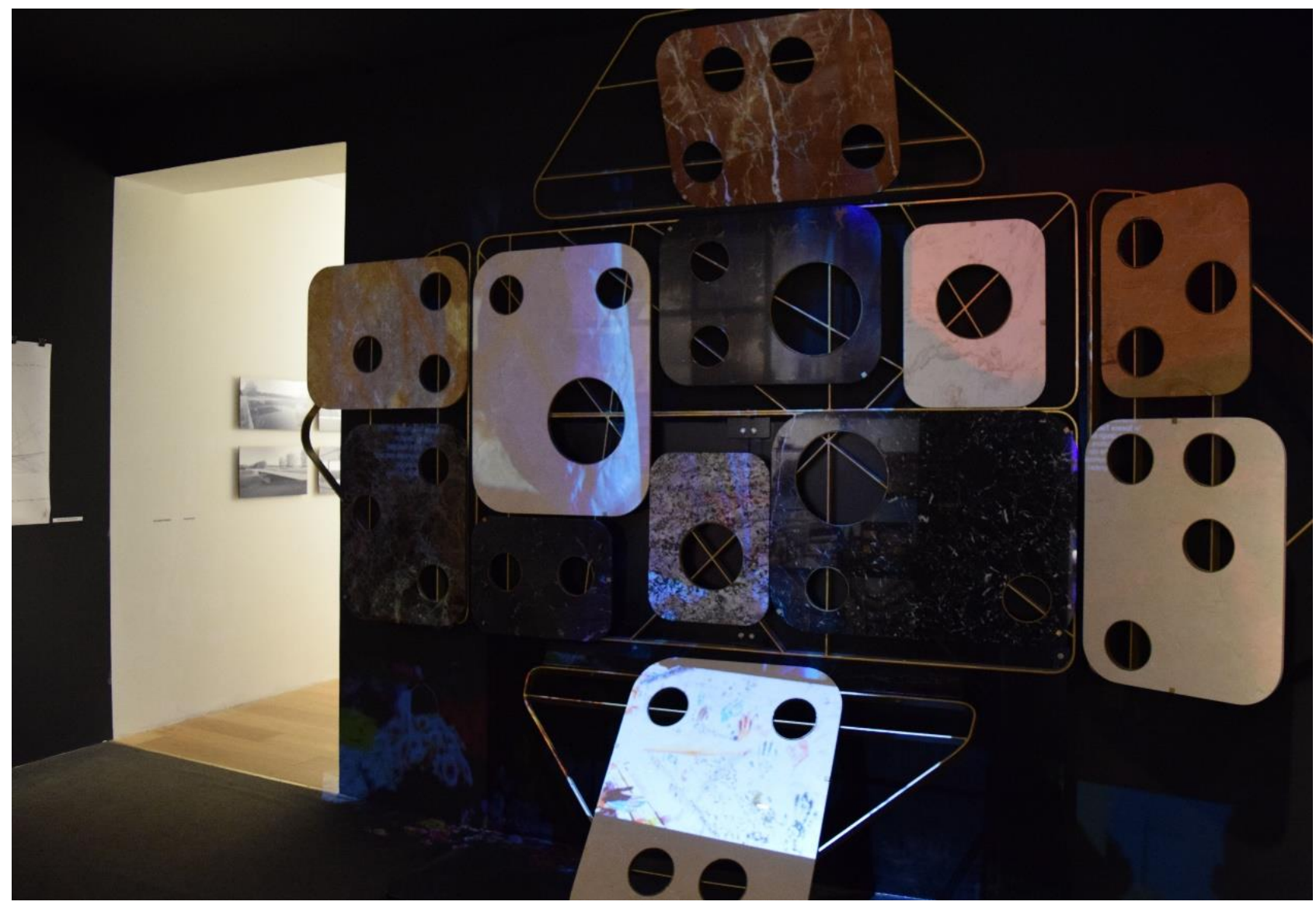

Fig. 06: Vista general del montaje de la instalación Dynamic Cities de Joaquín Alvado Bañón 


\section{El contexto de la instalación: en los márgenes Unfinished}

Los montajes desplegados en las tres sedes del European Cultural Center, donde se encuentra Dynamic Cities, son una extensión de los recintos de la propia Bienal de Venecia: el Arsenal y los Jardines; todo ello dentro de la propia ciudad, Venecia, algo más sólida y permanente a pesar de las continuas 'invasiones bárbaras' de hordas de turistas que parecen hundirla poco a poco a su paso. Así pues, procede un comentario sucinto a las muestras de los pabellones y, en particular, al Pabellón de España - Unfinished- que resultó ganador del concurso de Exposiciones y cuyo reconocimiento pone de manifiesto el buen estado de la crítica de arquitectura española y permite insertar el trabajo del profesor Alvado en el contexto del evento y las propuestas de los distintos equipos multidisciplinares en representación de todas las instituciones participantes.

Dentro del Jardín de la Bienal, sito en la punta Este del conglomerado de islas que conforman Venecia, se encuentra una serie de pabellones de diversos países. Un rápido recorrido por sus contenidos nos indica la adecuación de muchas de las temáticas abordadas a los objetivos marcados para esta $15^{\mathrm{a}}$ edición por el director y comisario de la misma, el arquitecto chileno, reciente Premio Pritzker, Alejandro Aravena. Estas metas se resumen en las palabras de este profesional bajo el lema: "Reportando desde el frente" y donde "propone compartir con un público amplio el trabajo de personas que escudriñan el horizonte a la búsqueda de nuevos campos de acción, enfrentando problemáticas sociales y urbanas como segregación, desigualdades, periferias, falta de infraestructura sanitaria, desastres naturales, escasez de vivienda, migración, informalidad, crimen, tráfico, basura, polución y participación de comunidades. Presenta, a la vez, ejemplos en los que diferentes dimensiones se hallan sintetizadas integrando lo pragmático con lo existencial, relevancia y audacia, creatividad y sentido común". Se trata de una propuesta para trabajar en los márgenes, en las fronteras, en aquellos frentes de batalla de las ciudades y de las arquitecturas que sirven a lo próximo, lo cotidiano y a la escasez de recursos de todo tipo, intentando no ganar ninguna guerra, sino siendo útil a los distintos segmentos que integran las sociedades del mundo, sin exclusiones.

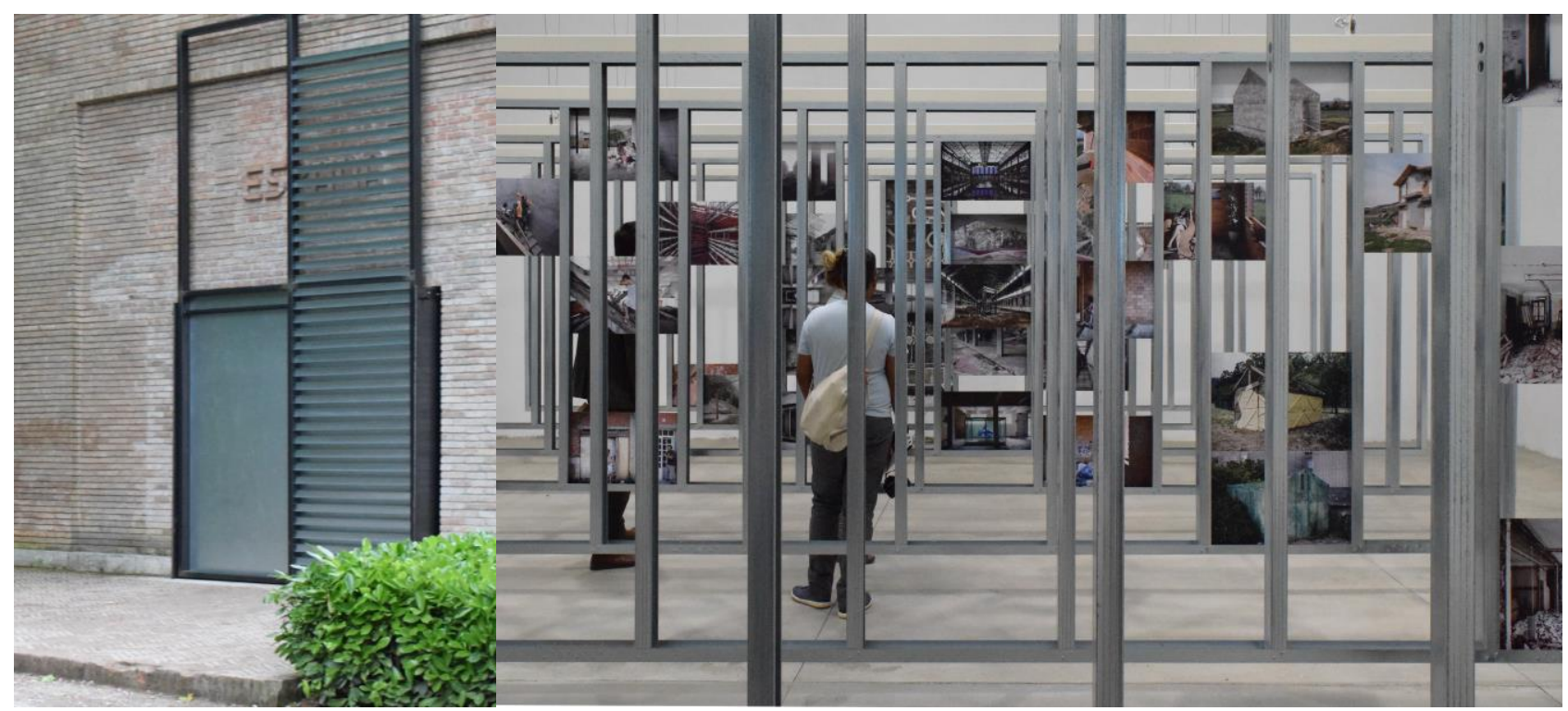

Fig. 07: Entrada al Pabellón de España y sala central (primera) con la colección de fotografías de los paisajes de la crisis

Del conjunto de Pabellones, conviene destacar algunos por su contenido experimental y de exploración de 'nuevos territorios'. El pabellón de Israel centra sus esfuerzos en mostrar una arquitectura colonizadora de lugares en condiciones extremas haciendo brotar la vida. Propuestas concretas de ayuda a comunidades se descubren en el pabellón Azul que aglutina a países de África. El pabellón-tienda del pueblo saharaui extiende cartografías de diversos campamentos al borde del desierto. El pabellón de Egipto realiza un gran despliegue sobre las condiciones de construcción de sus metrópolis (El Cairo, Alejandría) y sobre alternativas de explotación sostenible de sus recursos. También el pabellón de Venecia investiga sobre las condiciones de contaminación de los territorios y ciudades que rodean la laguna donde se inserta la propia ciudad ofreciendo ya un programa alternativo de reciclaje de residuos y plantas ecológicas que está en funcionamiento. El pabellón de Venezuela expone un variado elenco de pequeños equipamientos públicos insertados en barrios de asentamientos informales, técnica conocida como 'Acupuntura Urbana': pequeñas acciones que pueden desencadenar grandes resultados. El recinto de Bélgica investiga en las obras pequeñas y los materiales reciclados en las viviendas obreras y de las periferias residenciales. Mientras el pabellón de Corea realiza un exhaustivo estudio de la evolución de las tipologías residenciales a lo largo del siglo XX en Seúl para indagar en las alternativas tras los cambios sociales, el pabellón de Japón 
explora territorios de lo doméstico mediante mínimos hábitats que se insertan en diminutos bloques que atesoran espacios de convivencia lúdica compartida. Por último, mientras el pabellón de los Estados Unidos estudia el caso de la ciudad de Detroit, cuna de las grandes empresas de la automoción, ofreciendo proyectos y programas de rehabilitación urbana, especialmente del centro con equipamientos públicos y privados, el pabellón de Rusia exhibe la recuperación del patrimonio arquitectónico del recinto de una de las grandes Ferias del periodo de la Guerra Fría en Moscú... Así pues, la directriz de los contenidos de esta Bienal rastrea los bordes difusos de la disciplina o, mejor aún, del campo de conocimiento y experiencias que es la arquitectura, a la vez que los formatos expositivos son muy variados: maquetas, mobiliario, fotografías, dibujos, gráficos, virtualidad, recorridos, pantallas, etc.

Una mirada detenida se merece el pabellón de España (Fig. 07) que recibió la distinción del León de Oro, un edificio de una sola planta acotada por un recio muro perimetral que encierra seis salas con iluminación cenital natural y blanca. El acceso se realiza por un gran hueco situado a eje de simetría que, para esta ocasión, se cerraba con una puerta que, al desplegarse, se convertía en la marquesina de bienvenida al interior. Dentro destaca la sala principal (Fig. 09) donde, sobre una serie de planos paralelos verticales, se despliega una serie de fotografías organizadas por temas Unfinished (urbanizaciones, edificaciones, obras excesivas, etc.) que muestran los restos de los efectos de 'la crisis del ladrillo', una crisis (quizás síntoma de otra sistémica) que no solo ha afectado a España, pero que aquí, sus efectos han sido devastadores. Como respuesta a las preguntas del desastre de este epicentro, basculan a su alrededor las otras cinco salas donde se intenta dar respuesta arquitectónica a esta coyuntura desde la escala humana, la escasez de medios, lo modesto, lo próximo y lo efímero. Cinco salas que contenían los trabajos de equipos y colectivos de arquitectos agrupando temáticas. Así aparecía una primera sala dedicada a la obra pública de limitado presupuesto, pero con fuerte impacto visual; una segunda de obras residenciales, domésticas y de interiores, casi bajo el lema de 'una casa dentro de la casa'; una tercera destinada a mostrar obras incompletas, parciales y fragmentarias; una cuarta que exhibía obras que son ejemplos de experimentación con nuevos materiales, o su reciclaje, y con limitados medios.

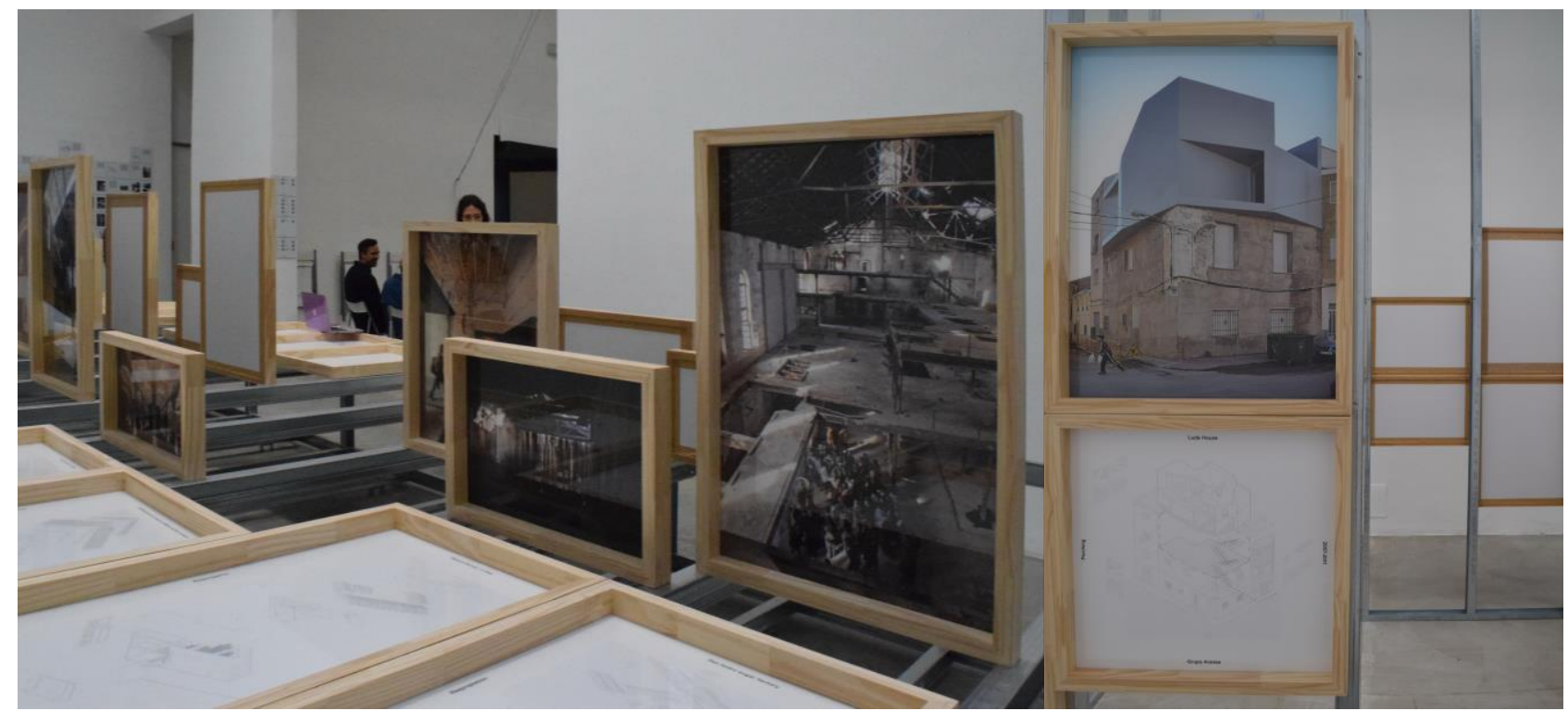

Fig. 08: Salas interiors del Pabellón de España con muestras de arquitecturas en tiempos de crisis y Casa Lude de F. Leiva

Todas estas salas se rematan con la quinta y última, sita tras la galería de exposición fotográfica del país tras el desastre inmobiliario donde, en multitud de pantallas, se proyectan audiovisuales con las opiniones de distintos arquitectos de fuera de España sobre la evolución y situación de la arquitectura española post-crisis. Entre las más de cincuenta obras de autor o en equipo que desfilan por las salas del pabellón, conviene citar la Casa Lude (Fig. 08), del arquitecto y profesor de la Universidad de Alicante, Francisco Leiva y su equipo Aranea. Se trata de una obra mínima, de elevación de poco más de una planta sobre un inmueble entre medianeras en la localidad de Cehegín (Murcia); una obra que ha sido objeto de numerosos reportajes y publicaciones en revistas, blogs y webs especializadas. Conviene señalar también que, igual que este caso, muchas de las obras seleccionadas, dado el periodo abarcado próximo a una década, resultan familiares porque ya han aparecido en diversos medios de comunicación e información profesional. 
Para concluir, siendo conscientes de los retos a los que se enfrenta la arquitectura, los profesionales y los estudiantes, como agentes mediadores de los procesos de diseño, definición y ejecución de las construcciones, del espacio público y, por ende, de nuestras ciudades, conviene una reflexión a propósito del tema planteado en $D y$ namic Cities y que se amplía en el seno de Reporting from the Front. ¿Seremos capaces de redistribuir la riqueza entre el norte y el sur, entre las ciudades opulentas del mundo informatizado y las metropolis suburbiales del planeta colonizado con el fin de reequilibrar la extracción de recursos con la abusiva producción de residuos y hacer de las ciudades lugares mejores donde convivir? Parte de la respuesta quizás se encuentre en esta exploración de los territorios que tiene aún que conquistar la arquitectura, una arquitectura entendida más como campo de conocimiento y como conjunto de saberes que no solo se nutren del mundo objetivo de la ciencia, sino que ha de asumir otros saberes que proceden de otras experiencias humanas. La arquitectura no está agotada, como tampoco lo están las ciudades que conforma, porque la humanidad está optando, desde la revolución industrial, por reunirse en las metropolis, por ser más urbana que rural, y aquí queda mucho por trabajar. Hay, pues, una invitación a transitar los márgenes y las fronteras de lo que se ha considerado hasta ahora la arquitectura para poner en crisis los conceptos tradicionales de la misma y para refundarla de nuevo atenta a las condiciones reales de las sociedades y al medio urbano y rural donde se desarrollan, tratando de establecer un equilibrio con la huella ecológica que nuestro paso deja sobre la Tierra. Una invitación a reflexionar sobre lo social y lo urbano a la vez a través de la arquitectura que está Unfinished.

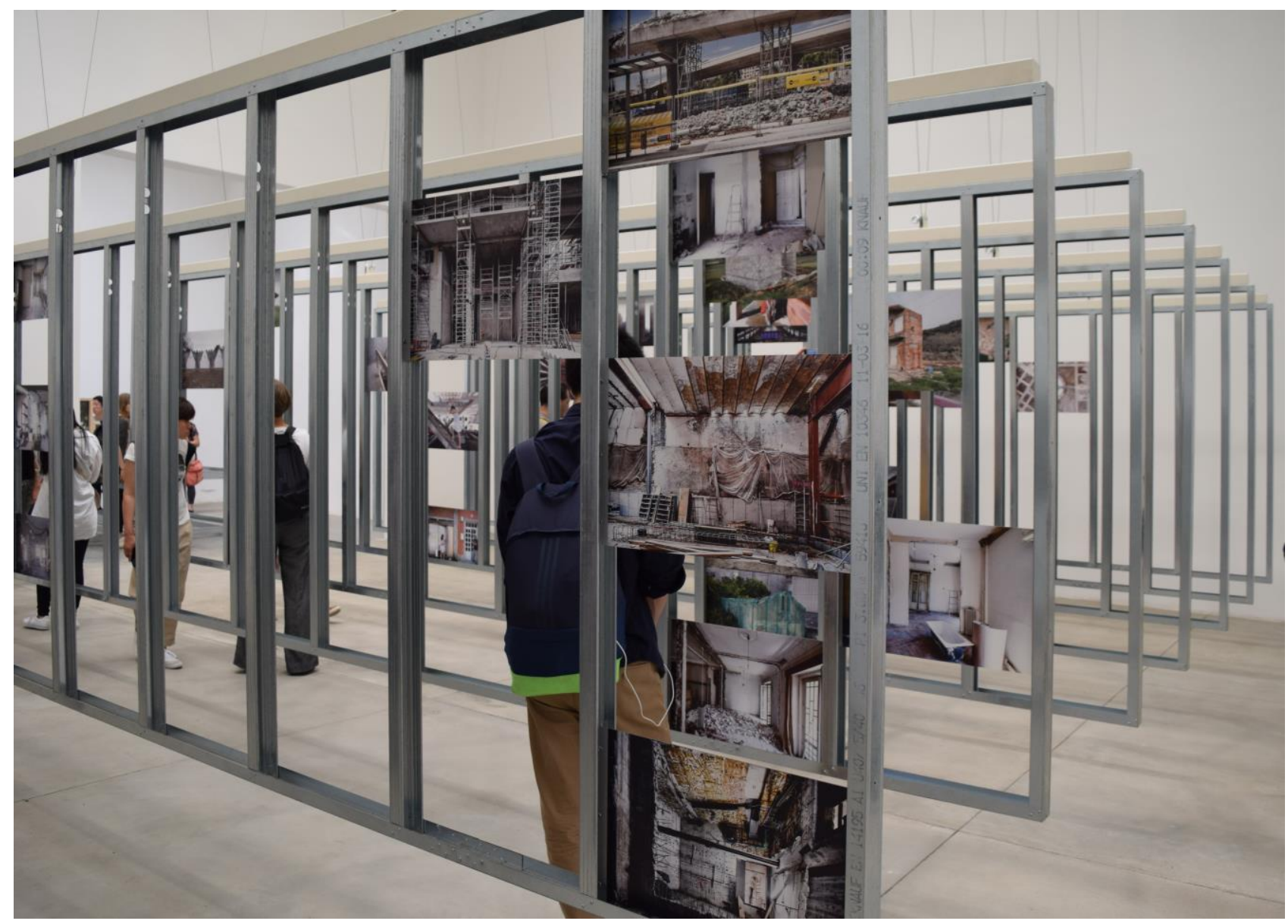

Fig. 09: Sala central del Pabellón de España con la colección de fotografías de los paisajes de la crisis del ladrillo $2007-2016$ 


\section{Bibliografía (para ampliación)}

-En relación a la 15를 Bienal de Arquitectura de Venecia:

http://www.labiennale.org/it/Home.html

https://www.google.com/culturalinstitute/beta/u/0/project/biennale-architettura-2016

http://www.plataformaarquitectura.cl/cl/tag/bienal-de-venecia-2016

-En relación a Alejandro Aravena, comisario de la Bienal:

AA.VV., "Elemental. Alejandro Aravena", rev. Monografías, no 185, 2016

http://www.plataformaarquitectura.cl/cl/795389/el-batallon-de-aravena-reportando-desde-el-frente

http://alejandroaravena.com/

-En relación al Pabellón de España Unfinished:

http://www.fomento.es/VIVIENDA/Bienal/Venecia/Obras presentaciondefiniTIVA.pdf

http://www.fomento.es/VIVIENDA/Bienal/Venecia/Pabell\%C3\%B3n\%20Espa\%C3\%B1ol.pdf

http://fundacion.arquia.es/es/ediciones/publicaciones/Colecciones/DetallePublicacion/123?coleccion=4

-En relación a la instalación de Joaquín Alvado:

AA.VV., Time, Space, Existence. La Biennale di Venezia 2016., ed. Global Art Affairs Foundation i.c.w. European Cultural Center, Italia, 2016, isbn: 978-94-90784-22-5

http://www.europeanculturalcentre.eu/index.php?page=45\&lang=en

https://web.ua.es/es/actualidad-universitaria/2016/abril16/1-10/joaquin-alvado-arquitecto-y-profesor-de-la-universidad-de-alicante-invitado-a-la-bienal-de-venecia-de-arquitectura-2016.html

http://www.diarioinformacion.com/cultura/2016/05/07/joaquin-alvado-avance-sociedad-pasa/1758724.html

https://joaquinalvado.wordpress.com/

-En relación a la Casa Lude de Francisco Leiva:

http://grupoaranea.net/blog/

-Fotografías que se acompañan: propiedad, ejecución y tratamiento del autor del artículo. 